Cinémas

Revue d'études cinématographiques

Journal of Film Studies

\title{
Un cinéma soufi? Islam, ombres, modernité
}

\section{Walid El Khachab}

Volume 11, numéro 1, automne 2000

Écritures dans les cinémas d’Afrique noire

URI : https://id.erudit.org/iderudit/024838ar

DOI : https://doi.org/10.7202/024838ar

Aller au sommaire du numéro

\section{Éditeur(s)}

Cinémas

\section{ISSN}

1181-6945 (imprimé)

1705-6500 (numérique)

Découvrir la revue

Citer cet article

El Khachab, W. (2000). Un cinéma soufi? Islam, ombres, modernité. Cinémas, 11(1), 133-149. https://doi.org/10.7202/024838ar

\section{Résumé de l'article}

Dans sa Poétique du cinéma, Raoul Ruiz cite l'émir Abdel Kader, qui utilise la figure de l'appareil photographique comme allégorie du rapport entre Dieu et les créatures : Dieu est invisible, mais la reproduction de ce négatif initial qu'est son image constitue les créatures et le papier des photos reproduites (Materia prima) correspond à la disponibilité du possible. Selon ses propres termes, Raoul Ruiz fait une lecture oblique d'Abdel Kader : en partant d'une perspective néoplatonicienne, il confine les réflexions de l'émir au statut des ombres projetées sur l'image (de Dieu) dans leur rapport avec la réalité. Ma lecture, peut-être oblique elle aussi, prétend jeter les bases d'une théorie fondée sur la dimension politique du soufisme et la conception soufie des ombres chinoises, qui pourrait expliquer le statut du cinéma et son rapport intrinsèque avec le mélodrame dans les pays de culture islamique. 


\section{Un cinéma soufi? Islam, ombres, modernité}

\section{Walid El Khachab}

\section{RÉSUMÉ}

Dans sa Poétique du cinéma, Raoul Ruiz cite l'émir Abdel Kader, qui utilise la figure de l'appareil photographique comme allégorie du rapport entre Dieu et les créatures: Dieu est invisible, mais la reproduction de ce négatif initial qu'est son image constitue les créatures et le papier des photos reproduites (Materia prima) correspond a la disponibilité du possible. Selon ses propres termes, Raoul Ruiz fait une lecture oblique d'Abdel Kader: en partant d'une perspective néoplatonicienne, il confine les réflexions de l'émir au statut des ombres projetées sur l'image (de Dieu) dans leur rapport avec la réalité. Ma lecture, peut-être oblique elle aussi, prétend jeter les bases d'une théorie fondée sur la dimension politique du soufisme et la conception soufie des ombres chinoises, qui pourrait expliquer le statut du cinéma et son rapport intrinsèque avec le mélodrame dans les pays de culture islamique.

\section{ABSTRACT}

In his Poétique du cinéma, Raul Ruiz quotes the emir Abdel Kader, who uses the figure of the camera as an allegory of the relationship between God and the creatures. God is invisible, yet the reproduction of this initial negative which his image is constitutes the creatures, while the paper of the reproduced pictures (Materia Prima) corresponds to the availability of the possible. In his own words, Ruiz reads Abdel Kader obliquely: starting from a neo-platonician perspective, he confines the emir's thoughts to the status of the shadows cast on the image (of God) in their relationship 
with reality. My reading, may be oblique as well, aims to lay the bases of a theory founded on the political dimension of Sufism and the Sufi conception underlying shadowshows, which would account for the status of cinema and its intrinsic relationship with melodrama in Islamic countries.

C'est probablement Raoul Ruiz qui, le premier, a relevé la pertinence de l'héritage soufi pour une réflexion sur le cinéma. Dans sa Poétique du cinéma, il cite l'émir Abdel Kader et évoque également Hallaj, Ibn Arabi et Ibn Toufayl. Pour ma part, je pense qu'une réflexion portant sur l'ancienne tradition soufie, qui affichait un intérêt marqué pour les ombres chinoises, pourrait jeter les bases d'une théorie mystique musulmane du cinéma. La portée de cette théorie apparaît certaine quand on replace le soufisme dans son contexte d'émergence.

Mon investigation part d'une interprétation politique du mouvement soufi. Je ne m'attarde donc pas sur l'aspect spirituel du soufisme et je m'écarte du débat qui occupe l'islamologie. Ce qui paraît fondamental dans le soufisme à ses débuts, au IX siècle, c'est qu'il constitue une nouvelle théorie de la médiation entre l'homme et Dieu. En proclamant que l'homme pouvait connaître Dieu et obtenir sa grâce sans passer par les prescriptions de la loi islamique, les soufis posaient un geste subversif par rapport à l'État. En effet, l'État était fondé sur un appareil de lois édictées à partir du livre sacré, le Coran, et s'inscrivant dans la tradition du prophète fondateur. Pour les docteurs de la loi, le salut de l'homme passait par l'adhésion à cette législation et, partant, à l'autorité de l'État. En ouvrant vers Dieu une voie sans passage obligé, une voie qui rend caduque la médiation du couple formé par la loi religieuse et l'État, les soufis se lançaient dans une entreprise anarchiste autant que mystique. C'est sans doute cela qui a valu sa crucifixion à Hallaj, et non pas le simple blasphème à propos de son union avec Dieu (Amin, 1992, p. 71-90 et Vitray-Meyerovitch, 1978).

Sachant que les soufis se sont intéressés au théâtre d'ombres au moins depuis le XII" siècle, on peut prétendre que cet ancêtre du cinéma était historiquement lié à ceux qui demeuraient à l'extérieur du territoire étatique et du paradigme du pouvoir. 
Mais leur réflexion sur les ombres et l'écran ne remettait pas en cause le seul pouvoir étatique. Elle minait la conception orthodoxe même de la transcendance.

\section{Cinéma et mimesis}

Un des intérêts majeurs de la théorie soufie des ombres chinoises est qu'elle permet d'échapper à la logique mimétique occidentale. Chez les soufis, les ombres chinoises ne sont pas le reflet du monde; elles sont un monde à part. Les penseurs arabo-musulmans ont connu Platon et Aristote. Mais, vers le XII ou le XIII ${ }^{e}$ siècle, la pensée grecque a fini par être rejetée de la production théorique musulmane. Il n'était plus question désormais de penser le monde comme ombre et reflet des Idées sauf chez quelques soufis, dont Ibn Arabi, pour qui l'homme est le reflet de Dieu. Il n'était non plus question de penser le monde comme un paradigme d'objets nommés par un paradigme correspondant, celui de la langue, ni de fonder la poésie sur la $m i$ mesis.

Depuis le IX ${ }^{e}$ siècle, le recours aux Grecs suivait un processus d'appropriation utilitaire de la culture par les Arabes vainqueurs, similaire au processus qu'avait connu la culture grecque dans l'empire romain naissant. Mais portant toujours la marque suspecte de la raison, dans une société où la légitimité du pouvoir est fondée sur la révélation et la loi religieuse, la philosophie et même la théologie basées sur un appareil logique et scolastique, le kalam, ont fini par être marginalisées.

Ainsi, la théorie soufie des ombres naît d'une veine populaire, départie de l'influence de la culture, et incompatible avec la structure épistémologique de domination du monde, que ce soit par la raison et les idées ou par le logos et la langue. Les écrits des soufis renvoient aux ombres comme allégorie du caractère illusoire du monde, par opposition à la vérité absolue de Dieu, mais n'établissent aucun rapport réflexif entre les ombres et le monde, même chez ceux qui estiment que le monde est le miroir de Dieu. On dirait que les mystiques étaient conscients de la structure de pouvoir sous-jacente à l'image de l'ombre. En effet, ils se démarquaient de l'idée du Calife ombre de Dieu sur terre, idée dominante dans la production théorique musulmane orthodoxe. 


\section{Cinéma et mysticisme}

Raoul Ruiz cite l'émir Abdel Kader qui utilise la figure de l'appareil photographique comme allégorie du rapport entre Dieu et les créatures: Dieu est invisible, mais la reproduction de ce négatif initial qu'est son image constitue les créatures et le papier des photos reproduites (Materia prima) correspond à la disponibilité du possible (Ruiz, 1995, p. 61).

Selon ses propres termes, Raoul Ruiz fait une lecture oblique d'Abdel Kader. En partant d'une perspective néoplatonicienne, il confine les réflexions de l'émir au statut des ombres projetées sur l'image (de Dieu) dans leur rapport avec la réalité. Il conclut que nous appartenons à un monde d'ombres et que l'image comprend des corps éclairés mais invisibles, simulacres du monde hermétique, sans rapport avec le monde réel des ombres. Pour lui, le réel est donc le monde des ombres, alors que le cinéma est celui des figures invisibles représentant des figures absentes, mais comprenant aussi des ombres réelles.

Raoul Ruiz se servait de bribes de considérations soufies et autres pour arriver à ses propres conclusions. Pour lui, l'image dans le cinéma comprend un inconscient photographique, notion plus étendue que celle conçue par Walter Benjamin (Ruiz, 1995, p. 55). L'inconscient photographique de Ruiz est une réverbération entre figures lumineuses et zones d'ombre, entre ce qu'il appelle particules images et anti-images (p. 60). Après avoir posé cet inconscient, il en arrive à la conception du cinéma qu'il souhaite défendre, résumée par le titre de son cinquième chapitre : "Pour un cinéma chamanique". Le cinéma est un univers où se côtoient hommes et fantômes, où s'effectue donc le contact entre le monde et l'au-delà. C'est le moyen de lire le monde qui est un livre de Dieu, et cette lecture se ferait en montrant les différentes enveloppes spirituelles qui entourent l'homme. Ruiz fait ici référence à deux autres mystiques musulmans: Ibn Toufayl et Hallaj (p. 71, 87-88).

La lecture de Ruiz combine des présupposés néoplatoniciens et une thématique du réel magique. Il reprend ainsi une longue tradition orientaliste qui ne lit le soufisme qu'à la lumière du néoplatonisme. Ce que je me propose, c'est de prendre Abdel Kader comme point de départ pour explorer les écrits soufis sur 
les ombres chinoises et tenter d'en extraire les paramètres de ce qui pourrait être une théorie mystique musulmane du cinéma. L'allégorie de l'appareil photographique chez l'émir soufi montre bien que le mysticisme musulman s'était ouvert à la venue, à l'action de la technique dans le monde et que le cinéma aurait pu constituer un nouvel enjeu de la réflexion soufie.

Ma lecture sera peut-être oblique, elle aussi, mais elle prétend jeter les bases d'une théorie offrant une issue à la théorie du mimétique qui s'est déployée avec l'expansion du cinéma. Étant donné que le cinéma des premiers temps dans le monde musulman était assimilé aux ombres chinoises, il est légitime de projeter les réflexions soufies sur les ombres en une théorie du cinéma. Cette théorie pourrait expliquer le statut du cinéma et son rapport intrinsèque avec le mélodrame dans les pays de culture islamique, vu la prédominance d'éléments mélodramatiques dans le théâtre d'ombres musulman. Ainsi, ce cheminement permettrait de spéculer sur ce qu'aurait pu être le cinéma dans cette culture, si son introduction n'avait été accompagnée de théories importées sur le statut mimétique des objets culturels et sur le rôle social de l'art.

Revenons à Abdel Kader et à l'allégorie de la photographie du roi, image du roi des cieux.

Nous appellerons l'image du roi voilé "distinction originelle de la réalité musulmane", Réalité des réalités, Unité Absolue, donnée originelle du Tout, etc. Quant au négatif initial, nous lui donnerons le nom d'Intellect Premier - première Forme spirituelle découlant de l'Être, son Kalam le plus haut, l'Âme Universelle. La reproduction de ce négatif constituera les genres et les espèces qui se propagent à travers le monde, et le papier urilisé pour les copies, Essence Immuable (la hylè grecque) $[s i c]$, Disponibilité du Possible (Ruiz, p. 61) ${ }^{\text {. }}$

Plutôt que néoplatonicienne, cette allégorie me paraît panthéiste. Il est vrai que, pour Abdel Kader, toutes les créatures "émanent» d'une source transcendante, mais leur essence n'est que matière: le papier. Ce qui est plus important, c'est que l'âme universelle est elle aussi matérielle: le négatif de même que la réalité des réalités sont l'image du roi et non le roi 
lui-même, ou son essence éthérée. Chez Abdel Kader, comme chez les soufis "radicaux", la transcendance n'est qu'une image. Je prétends que pour eux l'ontologie divine n'est pas reléguée à une sphère hermétique, comme le présume Raoul Ruiz, mais se trouve tout simplement écartée. La théorie soufie des ombres a ceci de "moderne" qu'elle place la transcendance dans l'immanence du monde et de la matière, comme la modernité a remplacé la transcendance divine par la raison, qui appartient à ce bas monde.

\section{Cinéma immanent}

Si Gilles Deleuze refuse de considérer les ombres chinoises comme faisant partie de la préhistoire du cinéma, c'est parce que l'invention des frères Lumière appartient au dispositif de la modernité. Pour lui, le cinéma est un système qui reproduit le mouvement à partir de moments quelconques immanents (les instantanés ou les photogrammes) plutôt que d'éléments formels privilégiés et transcendants (les formes de l'Antiquité ou les poses). Le cinéma rejoint ainsi l'astronomie moderne de Kepler et la physique moderne de Galilée, qui rapportent elles aussi le mouvement à une succession mécanique d'instants chronométrables et similaires: la première, en explorant la relation entre l'orbite et le temps mis à la parcourir; la seconde, en étudiant la relation entre le temps de la chute d'un corps et l'espace parcouru par ce corps (Deleuze, 1983, p. 13-14). Or, cette argumentation elle-même présuppose un rapport entre la modernité sécularisante et la mise à l'écart de la transcendance. Mon propos n'est pas de tenter de détecter une modernité préfigurée par les ombres chinoises, mais de repérer les éléments qui me semblent fructueux dans la conception soufie de ces ombres.

Des mystiques comme Ibn Arabi - évoqué d'ailleurs par Raoul Ruiz - ont estimé que l'écran cachant le montreur qui manipule les ombres est l'allégorie du voile cachant Dieu, cause première et moteur de la création (Kayyal, 1995, p. 25; Addas). Mais je voudrais donner une interprétation plus radicale de la veine mystique que j'explore, grâce au poète mystique Ibn al-Faridh:

[...] for in illusion's sleep / The shadow-phantom's spectre brings to thee / That the translucent curtains 
do reveal. / Thou seest forms of things in every garb / Displayed before thee from behind the veil / Of ambiguity: the opposites / In them united for a purpose wise: / Their shapes appear in each and every guise: / Silent, they utter speech: though still, they move / Themselves unluminous, they scatter light (Arberry, 1952, p. 68).

Ibn al-Faridh finira par dire que l'écran cache le montreur d'ombres, c'est-à-dire la transcendance. Mais le poète mystique dit également que l'écran révèle l'ambiguïté, celle d'objets à la fois silencieux et parlants, immobiles et mobiles, non lumineux et éclairants. Le cinéma n'est-il pas au cœur même de cette problématique de l'ambiguïté ? N'est-il pas cet entre-deux, entre l'instantané et l'illusion de mouvement produite par la machine à projection? Plus loin, Ibn al-Faridh compare les ombres au chant des oiseaux émouvant les hommes et produisant néanmoins un "discours non interprétable". Le cinéma n'est-il pas, entre autres, la trace de l'indicible dans l'image? Cet indicible, inconvertible en mots, et produisant pourtant une médiation signalée par l'émotion, n'est-ce pas une forme d'inconscient cinématographique? La révélation dans les vers d'Ibn al-Faridh n'est pas celle d'une transcendance située dans une sphère sublime et éthérée. Le "lieu» de la transcendance serait lié à l'ambiguïté, donc situé à la fois derrière et dans l'écran, ou plus précisément dans la transparence de l'écran, dans cet entre-deux situé entre les marionnettes et le tissu tendu sur son châssis.

Je tiens à prouver la "sécularité" de l'écran des ombres chinoises chez les soufis parce qu'elle est inséparable du rôle politique de cette pratique culturelle. Une nouvelle lecture des mystiques musulmans nouls autorise à avancer que, pour certains d'entre eux, le spectacle d'ombres est plus qu'une parabole révélant l'omniprésence de Dieu et l'orientation du monde immanent vers une transcendance dans l'au-delà. Les ombres, plutôt que de doubler la soumission de ce monde à l'autorité transcendantale du Prince, constituent un principe agissant. Elles sont un mouvement à l'œuvre dans le monde, une "agentivité" (agency). L'essentiel des vers consacrés aux ombres dans le poème d'Ibn alFaridh décrit ce foisonnement du monde des ombres. L'action 
en question peut être "militante" - comme on le voit dans les spectacles d'ombres ridiculisant les soldats français pendant l'occupation du Maghreb (Kayyal, 1995, p. 25). Mais sa valeur peut résider dans le fait qu'elle est action, créant par exemple un espace pour des pratiques s'opposant à l'immobilité de l'ordre établi, donc marginalisées par l'État.

\section{Cinéma et devenir}

La réflexion de Deleuze sur le cinéma présuppose que celui-ci relève du paradigme du mouvement: un mouvement séculaire et immanent, qui n'est pas tributaire d'un moteur divin. La théorie musulmane des ombres présente une certaine analogie avec cette idée. Les ombres y sont inscrites dans une conscience du mouvement et du devenir, même si ce mouvement est parfois compris comme cyclique ou linéaire. Les musulmans concevaient les ombres chinoises comme figure du passage du temps. $\mathrm{Au} \mathrm{X}^{\mathrm{e}}$ siècle, le jurisconsulte andalou Ibn Hazm comparait la succession des générations aux ombres successives projetées sur l'écran à partir d'une roue portant des images. C'est là une des premières allusions à un spectacle d'ombres dans le monde islamique. Rien de prémoderne dans cette vision cyclique du temps. Mais déjà au $X V^{e}$ siècle, le poète égyptien Ibn Sudun comparait les vicissitudes du temps aux ombres chinoises (Moreh, 1992, p. 73 et 123).

Cette dynamique explique en quoi les ombres sont épistémologiquement subversives dans la culture islamique. Étant le lieu du devenir perpétuel, l'image des temps qui changent, elles s'opposent à la fixité de l'ordre établi. Très tôt dans l'histoire islamique, le Coran, littéralement "ce qu'on récite", est consigné dans un livre canonique, le Moshhaf, littéralement "le folio", et sert de référence à divers "codex ». Dans une culture dominée par l'immobilisme du Livre institutionnalisé, les ombres comme pratique du devenir s'avèrent rebelles.

Inscrites dans le devenir, lieu par excellence du multiple, les ombres, comme le cinéma, minent inévitablement les idées d'absolu et d'unicité des valeurs. Ibn Daniel, le patriarche des ombres dans la tradition musulmane, disait par la bouche de son montreur d'ombres que " underlying every shadow a truth is 
to be found" (Badawi, 1982, p. 92). Il est clair que cet énoncé s'oppose à cette idée des musulmans conciliateurs selon laquelle les ombres indiquant la présence du moteur divin, c'est-à-dire la vérité absolue. Je présume donc que la remarque d'Ibn Daniel ne signifie pas que les personnages des ombres sont "vrais", mais qu'elle indique l'évacuation de l'idée d'une seule vérité et prône la multiplicité, à l'instar de la multiplicité des ombres.

Le caractère subversif des ombres en a fait l'espace par excellence des pratiques culturelles marginales ou non orthodoxes. Les pièces d'ombres qui nous sont parvenues depuis le XII ${ }^{e}$ siècle montrent la place importante qu'y occupent, par exemple, les thèmes du libertinage et de l'homosexualité. On a pu expliquer la répression périodique qui s'est abattue sur les montreurs d'ombres, tout le long de l'histoire islamique, par l'audace de leurs thèmes, par la virulence avec laquelle ils critiquaient les représentants du pouvoir, ou encore, par l'interdiction de la figuration dans la tradition islamique orthodoxe, ou encore par l'audace des critiques faites des représentants du pouvoir (Moreh, 1992, p. 25). Je dirais que cette répression a une justification épistémologique: les ombres relèvent du paradigme du devenir par opposition au territoire de l'immobilisme; le spectacle d'ombres est une pratique d'outsiders, désabusés quant à la « médiation" de l'État. Cette hypothèse, me semble-t-il, explique mieux le fait que la répression frappait tous les montreurs d'ombres, sans se préoccuper d'épargner les «modérés».

C'est peut-être là l'origine de la sévérité de la censure du cinéma dans les pays musulmans dominés par la tradition orthodoxe. La censure a été importée avec le cinéma en terre d'Islam. Mais si, en Europe et en Amérique, elle a évolué tout le long du $\mathrm{XX}^{e}$ siècle dans le sens d'une tolérance croissante, la censure a rarement suivi ce mouvement dans les pays musulmans.

L'action politique des ombres - et du cinéma - va au-delà de la provocation. Elle réside dans sa propre dynamique, dans la pratique continuelle de la transformation et du multiple. Ibn alFaridh comme Ibn Arabi vénèrent la métamorphose. À travers l'univers soufi retentit le cri d'Ibn Arabi: "Mon cœur est capable de toutes les formes. " La transformation dans et par les ombres apparaît également dans des paradigmes : éveil et rêve, vie et 
mort. Ibn al-Faridh notait cette particularité de l'univers des ombres où la dynamique du "fantôme de l'ombre " prend place dans "l'illusion du sommeil" (Arberry, 1952, p. 68). Le poète mystique signale non seulement encore un entre-deux propre aux ombres et au cinéma, mais également une situation de passage continuel entre deux états. Il ne s'agit pas d'une évaluation négative assimilant les ombres à l'univers du rêve, opposé à l'univers positif de la réalité.

Ce genre d'oppositions, caractéristique de la modernité, mène souvent à des conclusions expéditives: par exemple, on condamne le cinéma parce qu'il est une usine à rêves, on s'en empare pour le confiner aux territoires non transgressifs du rêve. Or, Ibn al-Faridh fait l'éloge de l'ambiguïté. Il ne se limite pas à opposer au rêve une vision "positiviste" du réel et de sa "logique", bien au contraire. Pour lui, l'illogique peut être une révélation et devrait donc jouir d'une attention particulière: " [...] Beware: turn not / Thy back on every tinselled form or state / Illogical [...]» (Arberry, 1952, p. 68). Cette vision est plus dynamique que le positivisme moderniste séparant catégoriquement ce qui est logique et ce qui est illogique, le rêve et l'éveil, la fiction et le réel, l'actuel et le virtuel. La théorie du cinéma a longtemps souffert de ce manichéisme, alors que c'est le cinéma qui problématise, en pratique, les rapports entre les éléments de ces dichotomies. L'image au cinéma indique clairement ce "circuit" entre l'actuel et le virtuel (Deleuze, 1996).

Le potentiel transformationnel des ombres atteint son comble dans l'opposition vie/mort. À cet égard, il est un récit hautement significatif dans la mythologie turque, qui relate la naissance du théâtre d'ombres. Les deux personnages les plus populaires des ombres, Karakoz et Iwaz, auraient été à l'origine deux ouvriers travaillant sur le chantier d'une grande mosquée dédiée au sultan. Vu qu'ils dissipaient la concentration de leurs compères par leurs plaisanteries et leurs histoires, le gouverneur les a fait pendre. Puis, regrettant leur absence, il ordonna que soient fabriquées deux marionnettes à leur effigie et qu'elles soient mues entre une lanterne et un écran (Kayyal, 1995, p. 20).

Dans le mythe des origines se trouve déjà inscrite la notion de subversion par rapport à la double transcendance du pouvoir (la 
mosquée et le sultan), et on remarque que la continuité de cette résistance passe par la transformation continuelle (de la vie à la mort, de la mort à la vie sous forme d'ombre). Cette conception de l'image comme fantôme relève d'un champ de vitalité subversive et de transformation constante. Elle me paraît plus dynamique que la conception d'un Edgar Morin, par exemple, qui reconnaît la puissance et l'amortalité de l'image comme spectre - ombre - et souligne son potentiel transformationnel. Mais sa réflexion n'accorde aucune part à l'ombre comme résistance et demeure prisonnière de l'archétype du double, ce qui d'ailleurs prolonge un paradigme remontant à l'Antiquité grecque et cause de l'impasse qu'a connue la modernité au cinéma (Morin, 1956, p. 31-39). Cette impasse se résumerait en deux visions contradictoires du cinéma comme double ( $\mathrm{du}$ réel) : soit que le cinéma devrait être le reflet fidèle du réel, soit que le cinéma ne saurait être qu'illusion parce qu'il est double et non réel.

\section{Cinéma et médiation}

Les soufis concevaient la sphère des ombres chinoises comme un monde autonome, nettement démarqué du monde "réel ». Ibn al-Faridh évoque les silhouettes fantomatiques "[cowering] darkly in their dim domain" (Arberry, 1952, p. 68). Cette conception se retrouve dans la pratique populaire: les textes des pièces d'ombres comprennent des allusions métafictionnelles à l'écran, appelé tente. Parfois, un personnage hésite à effectuer telle ou telle action, attendant l'autorisation du personnage principal, Karakoz, "parce que, dit-il, la tente est celle de Karakoz" (Kayyal, 1995, p. 10). L'écran est donc un monde à part, gouverné par Karakoz et les autres ombres, et non par les lois du monde «réel».

La lecture d'Ibn al-Faridh révèle combien est important le rapport regardant/regardé qu'il instaure entre les hommes et les ombres, entre le monde et l'écran, rapport marqué par l'usage récurrent de l'apostrophe. Le poète enjoint constamment son auditeur, spectateur des ombres, de ne pas tourner le dos à l'écran, lui rappelle qu'il pleure ou rit à cause de ce qui est exposé devant lui, et utilise le champ lexical du regard (gaze, see, glimpse) six fois en soixante-trois lignes. La distinction entre les 
deux mondes est évidente. La dynamique du devenir crée un univers en constante transformation, mais qui trace des frontières nettes entre regardant et regardé.

Les ombres, et plus tard le cinéma, ont constitué dans le monde islamique un espace privilégié de médiation, et ce, suivant la tradition soufie, c'est-à-dire sans tenir compte de l'État comme médiateur entre l'homme et ses semblables, encore moins entre l'homme et Dieu. Les questions "préoccupantes" se négociaient au quotidien, hors de portée de l'appareil étatique, sur l'écran des ombres, d'où la place qu'y occupent l'allogène, le fantastique et l'occulte, l'illicite, l'immoralité, l'homosexualité, etc.

Depuis les premières projections de la fin du XIX siècle, le cinéma en terre islamique a été perçu comme un prolongement technique des ombres chinoises et de la lanterne magique. Dans les comptes rendus des premières projections cinématographiques en Égypte, on parle tantôt d'ombres et de fantômes, tantôt de films et d'images, et on fait parfois la comparaison entre les ombres chinoises et le cinématographe. Le terme "littéraire " désignant le cinéma en arabe, khayyala, est dérivé de la racine signifiant ombre, khayal (Chalach, 1986, p. 36-37; Hadari, 1989, p. 17, 24 et 38).

Un hasard historique a voulu que le mélodrame régnant sur le cinéma de fiction des premiers temps s'ancre dans la tradition des ombres de la culture islamique. Il est aisé de comprendre la "parenté" entre les deux pratiques culturelles. En effet, il se trouve que les ombres ont un cachet mélodramatique - prédominance des chants et de la musique, situations pathétiques, libertinage débouchant sur un repentir final, goût du spectacle: démons, monstres, combats, etc. (Badawi, 1982, p. 106). Depuis sa naissance au thêâtre, le mélodrame a accompagné la modernité européenne en tant que critique et espace de négociation de celle-ci. Le mélodrame cinématographique a été introduit dans la culture islamique avec les autres produits culturels et institutionnels de la modernité, importés depuis la fin du XVIII siècle par des despotes éclairés tels que les sultans réformateurs en Turquie, les vice-rois de la dynastie Mohamed Ali en Égypte et les Shahs de la dynastie Quajar en Iran. 
Il me semble que le mélodrame cinématographique, par rapport aux changements produisant la modernité en terre d'Islam, remplissait la même fonction de médiation que celle assumée traditionnellement par les ombres. C'est ce mélodrame qui montrait par exemple les vertus d'un État centralisé moderne faisant régner l'ordre perturbé momentanément par les nomades, rebelles à cette autorité (Baiser dans le désert, Égypte, 1927; La Fille lore ou Iran hier et aujourd'hui, Iran, 1932), ou encore, le respect de la tradition dans le village, opposé à l'occidentalisation de la ville (Zeinab, Égypte, 1930; L'Homme sensuel, Iran, 1933).

Cela explique peut-être le fait que la médiation de la modernité dans les pays musulmans s'effectuait en particulier dans le cinéma, et que les cinémas nationaux y étaient dominés par le mélodrame. Un survol rapide des débuts du cinéma en Égypte, en Turquie et en Iran confirme cette prédominance du mélo. On a même comparé le film iranien commercial typique au canon égyptien (Boughedir). On pourrait justifier la prédominance du mélo en terre d'Islam par le fait que ce marché était dominé par des mélodrames européens et hollywoodiens importés. Mais celle-ci est mieux expliquée, quand on cherche l'origine de la théâtralité de l'excès mélodramatique ainsi que du recours à de "grosses ficelles", par la tradition du théâtre d'ombres.

Mon hypothèse est que le cinéma, nouvellement arrivé en terre d'Islam, jouissait du même statut épistémologique que les ombres. Mais avec le raffermissement du processus de modernisation par l'occidentalisation, l'importation du cinéma dans la culture islamique a été accompagnée d'une importation des théories mimétiques et de celles sur le rôle social de l'art. Ce qui a influé sur le mode de réception traditionnel des ombres qui servait au départ de modèle de consommation pour le cinéma. Dans une phase intermédiaire, le cinéma était sans doute pour le grand public un espace se dérobant à l'autorité directe de l'État, mais subissant l'influence des idéologies promues par celui-ci. Avec une plus grande assimilation de la modernisation, l'appareil bureaucratique et son pouvoir prenaient de l'ampleur et l'État réapparaissait comme l'instance primordiale de la 
médiation sociale. De même, les théories occidentales prenaient la valeur de normes de la civilisation. Le cinéma demeurait un lieu de divertissement, comme les ombres, mais n'était plus considéré comme non mimétique. Le discours culturel de la modernité dans le monde islamique fait du cinéma le porteur d'un message et d'une morale, et le considère comme le reflet d'une certaine réalité sociale. Ce revirement a eu lieu en deux décennies, peut-être. Tout se passe entre les premiers articles sur le cinéma perçu comme curiosité scientifique, comme divertissement étonnant à cause de l'illusion du mouvement qu'il donne, à la fin du XIX $X^{\mathrm{e}}$ siècle, et les articles attaquant le cinéma national parce qu'il montre des paysages misérables et des danses orientales, portant ainsi atteinte à l'image de la nation dans les forums étrangers, dans les années vingt $\mathrm{du} \mathrm{XX}^{\mathrm{e}}$ siècle. Les presses égyptienne et iranienne abondent en exemples qui permettent d'apprécier ce revirement ${ }^{2}$.

Il est sans doute plus juste, ou plus prudent, de présumer que, comme pratique culturelle de masse, le cinéma dans les pays musulmans demeurait au tournant du siècle une production abordée par une théorie hybride, mêlant l'acquis traditionnel et l'apport occidental. D'une part, c'était une sphère autonome, non mimétique, peuplée de traîtres, de victimes et de sauveurs, et d'autre part, c'était un produit où l'on cherchait à s'identifier comme réalité sociale.

\section{Limites du cinéma soufi}

Je pense que ce sont les insuffisances de la théorie de la médiation par les ombres chez les soufis qui rendaient la sphère culturelle musulmane réceptive aussi bien à l'étatisation ayant accompagné la modernisation qu'au détournement du potentiel subversif des ombres en faveur de formulations européennes d'une théorie du cinéma.

Les soufis concevaient la médiation par les ombres selon un schéma linéaire $:$ médiation = regardant + ombres (regardées) $\rightarrow$ révélation - que l'objet révélé soit le moteur premier ou l'idée d'ambiguïté. Cette vision linéaire et instrumentale de la médiation - même en évacuant le poids de l'appareil étatique rend facile le détournement du schéma, le transformant en 
quête de nouveaux éléments transcendantaux: plutôt que Dieu ou l'État, la nation ou le réalisme social. C'est cette substitution qu'ont connue les pays musulmans avec l'institutionnalisation du cinéma d'après le modèle occidental. Ainsi, au lieu d'être un espace du devenir transgressant la fixité, le cinéma s'est territorialisé dans le monde islamique selon les paradigmes modernistes du nationalisme et du réalisme.

Chez les soufis, les ombres chinoises semblent constituer un lieu privilégié de la médiation, à cause de l'importance accordée aux ombres en tant qu'objet regardé, à cause également du fait même de regarder. En dehors de leur théorie des ombres, les soufis concevaient le rapport entre l'homme et le divin comme une relation englobant tous les aspects de la vie de l'individu. Cependant, vu l'importance des ombres comme divertissement populaire, il fallait les cautionner par une couverture morale. Ainsi, les ombres préparaient déjà le terrain à une certaine conception du cinéma: le cinéma comme lieu privilégié d'un message à transmettre, de la concrétion d'un rapport privilégié avec le transcendantal. La dynamique de la médiation, insaisissable dans une simple équation, difficile à circonscrire précisément, se trouvait de la sorte oblitérée.

Ainsi, au XIX ${ }^{e}$ siècle, certains spectacles commençaient par l'intervention d'un assistant du montreur d'ombres, dit wassaf, "le descripteur ", qui rappelait que l'écran est le voile de la destinée et que Dieu a fait des ombres des paraboles pour que les hommes en tirent des leçons (Kayyal, 1995, p. 25-26). C'était sans doute un moyen de cautionner le spectacle, mais la transformation de cette pratique en tradition a dû habituer le public à attendre des ombres, et plus tard des images cinématographiques, un message; le théâtre d'ombres allait devenir, comme le cinéma, ce lieu d'une médiation entre le spectateur et le sens voulu par Dieu (ou par l'auteur, ou par l'État, dans le contexte de la modernité). Dans ce sens, plus que le montreur, le descripteur est l'ancêtre du bonimenteur; il est celui qui donne sens à l'image et essaye d'imposer un "ordre" à son chaos.

Lanalyse menée dans les pages précédentes se base sur une conception de la médiation considérée comme production sociale continuelle (Deleuze, 1974) et comme un processus dont 
les parties ne sont identifiables que schématiquement et a posteriori, à l'instar d'une réaction chimique. On sait qu'il y a échange d'électrons entre les corps, mais on ne peut pas repérer à l'œil nu les molécules qui participent à cet échange ni le moment précis où chaque molécule se transforme. La médiation se produit à chaque instant du vécu quotidien et ne se limite pas à un espace. Elle implique non pas un rapport dichotomique entre l'homme et le monde, ni entre deux parties bien distinctes et identifiées, mais plutôt une interaction dynamique entre les molécules peuplant l'univers. La médiation est ainsi inséparable de la matérialité: elle ne se produit pas entre le physique et le métaphysique; elle s'inscrit dans un processus de transformation continuelle ${ }^{3}$.

De ce point de vue, le cinéma n'est donc pas un espace privilégié de la médiation, mais un espace offrant des figures de la médiation. C'est une sphère où l'on peut prendre conscience du phénomène, non pas parce que celui-ci y est figuré, mais parce qu'il se produit constamment, dans la mesure où le film est une transformation-production de ce qui est situé devant l'objectif, ou imprimé par les techniques de l'image sur la pellicule, et dans la mesure où l'on est confronté à la matérialité de l'image, tout le long de la projection du film.

Cette critique de la théorie des ombres et de la médiation chez les soufis montre bien que leur "mysticisme matérialiste", pour ainsi dire, n'était pas une voie de salut barrée par la modernité et son idéologie de la représentation, dans laquelle le monde finit par devenir une représentation du sujet central et hégémonique (Heidegger). La théorie soufie constitue simplement une possibilité gommée par l'occidentalisation. Si l'histoire était à refaire, cette théorie aurait pu proposer le cinéma comme un modèle d'être dans le monde et une dynamique déstabilisant le schéma du contrôle de l'individu par l'État. Reste que le contexte médiéval de la production de cette théorie aurait également rendu nécessaire une critique des présupposés linéaires des soufis. 


\section{NOTES}

1. Voir également la traduction de Michel Chodkiewicz (Émir Abdel-Kader. Écrits spirituels, Paris: Seuil, 1982, p. 120).

2. Ali Issair maintenait encore cette norme du reflet du réel dans son histoire du cinéma iranien, Cinerna in Iran. 1900-1979 (Metuchen, New Jersey \& London: The Scarecrow Press, 1989). De même, Galal El Charkawi, dans sa thèse sur l'histoire du cinéma égyptien, Rissah fi tarikh al-cinéma al-misriah (Le Caire: Al-Charikah almisriah lil tiba'a wal nachr, 1970).

3. Cette analyse s'inspire largement du séminaire de Silvestra Mariniello portant sur la médiation audiovisuelle et tenu à l'Université de Montréal.

\section{OUVRAGES CITÉS}

Addas, Claude. Ibn Arabi ou la quête du soufre rouge. Paris: Gallimard, 1989.

Amin, Hussein Ahmed. Le Livre du musulman désemparé. Paris : La Découverte, 1992.

Arberry, Arthur J. The Poem of The Way. London : Emery Walker, 1952.

Badawi, M. M. «Medieval Arabic Drama: Ibn Daniyal ». Journal of Arabic Literature. Leiden : E. J. Brill, vol. 13, 1982.

Boughedir, Farid. "Le cinéma Motefavet iranien", dans Guy Hennebelle, Quinze ans de cinéma mondial, Paris : Cerf, 1976.

Chalach, Ali. Al-Naqd al-cinémaï fil sahbafa al-misriyah (La critique cinématographique dans la presse égyptienne). Le Caire: GEBO, 1986.

Deleuze, Gilles et Claire Parnet. Dialogues. Paris : Flammarion, 1996.

Deleuze, Gilles. L'Image-mouvement. Paris : Minuit, 1983.

Deleuze, Gilles. L'Anti-CEdipe. Paris: Minuit, 1974.

El Charkawi, Galal. Rissalah fi tarikh al-cinéma al-misriah (thèse de doctorat sur le cinéma égyptien). Le Caire: Al-Charikah al-misriah lil tiba'a wal nachr, 1970.

Hadari, Ahmed. Tarikh al-cinéma fi Misr (Histoire du cinéma en Égypte). Le Caire: Nadi al-cinéma bil-Cahira, 1989.

Issari, Ali. Cinema in Iran. 1900-1979. Metuchen, New Jersey \& London: The Scarecrow Press, 1989.

Kayyal, Mounir. Maßjam babat masrah al-Zhill (Dictionnaire du théâtre d'ombres). Beyrouth: Maktabat Lobnan Nacheroun, 1995.

Moreh, Shmuel. Live Theatre and Dramatic. Literature in The Medieval Arab World. New York: New York University Press, 1992.

Morin, Edgar. Le cinéma ou l'homme imaginaire. Paris: Minuit, 1956.

Ruiz, Raoul. Poétique du cinéma, tome I. Paris: Dis Voir, 1995.

Vitray-Meyerovitch, Eva. Anthologie du soufisme. Paris: Sindbad, 1978. 